\title{
Performance Evaluation of the Thick Pinhole Gamma Rays Diagnostic System*
}

\author{
Hongwei Xie, Jinchuan Chen, Linbo Li, Qiang Yi \\ Institute of Nuclear Physics and Chemistry, China Academy of Engineering Physics, Mianyang, China \\ Email: xiehw1966@sina.com
}

How to cite this paper: Xie, H.W., Chen, J.C., Li, L.B. and Yi, Q. (2018) Performance Evaluation of the Thick Pinhole Gamma Rays Diagnostic System. Journal of Analytical Sciences, Methods and Instrumentation, 8, 25-36

https://doi.org/10.4236/jasmi.2018.83003

Received: May 9, 2018

Accepted: September 4, 2018

Published: September 7, 2018

Copyright $\odot 2018$ by authors and Scientific Research Publishing Inc. This work is licensed under the Creative Commons Attribution International License (CC BY 4.0).

http://creativecommons.org/licenses/by/4.0/

\begin{abstract}
The diagnostic methods for the profile of the radiation source were established at first based on the pinhole imaging principle. In this paper, the relationships among various parameters of the gamma-rays crammer such as the modulation transfer function $(M T F)$, the noise power spectrum (NPS), the signal-noise ratio $(S N R)$ and the detective quantum efficiency (DQE) are developed and studied experimentally on the cobalt radiation source. The image diagnostic system is consisting with rays-fluorescence convertor (YAG crystal), optical imaging system, $M C P$ image intensifier, $C C D$ camera and other devices. The spatial resolution of the modulation transfer function $(M T F)$ at $10 \%$ intensity was measured as $1 \mathrm{lp} / \mathrm{mm}$ by knife-edge method. The quantum of the measurement system is about 150 under weak radiation condition due to the single particle detection efficiency of the system. The dynamic range was inferred preliminarily as about 437 . The required radiation intensity was calculated using the experiment result for the $S N R=1,5,10$, respectively. The theoretical investigation results show that the radiation image with $S N R$ $=1$ can be only obtained when the pinhole diameter is $0.7 \mathrm{~mm}$, object distance and image distance are both $200 \mathrm{~cm}$, and the radiation intensity is about $1.0 \times 10^{12} \mathrm{Sr}^{-1} \cdot \mathrm{cm}^{-2}$.
\end{abstract}

\section{Keywords}

Gamma-Ray Camera, Pinhole, YAG, $M C P$ Image Intensifier, $C C D, M T F$, NNPS

\section{Introduction}

The $\gamma$ (neutron)-rays camera based on the pinhole imaging principle are widely

*1) Sponsered by: National Natural Science Foundation of China (Contract No. 11005095, 11305155). 2) About the author: Hongwei Xie (1966), male, associate professor, master of science, mainly engaged in the study of image diagnostics of pulse radiation source. 
used in the diagnostics of the radiation sources [1]. The diagnostic method based on $M C P$ image intensifier, due to its performance in image intensification and shutter time gating, is also widely used in the study of imaging diagnostics of the pulse radiation source and weak radiation sources [2] [3]. The system consists of rays-fluorescence convertor (YAG crystal), optical imaging system, $M C P$ image intensifier, $C C D$ camera and other devices. Radiation imaging quality is not only dependent on the quality of the imaging system, but also on other various factors, e.g., the incident flux of the $\gamma$-rays, the detecting efficiency of the $\gamma$-rays, energy conversion efficiency and quantum gain of the photographic system, etc.

On the other hand, imaging based on radiation source combined with thick pinhole is an ideal method of nondestructive detection with high spatial resolution. Point radiation source is ideal to be used in the nondestructive detection as the size of the radiation source is the critical factor for the spatial resolution, while it's challenging to decrease the source size during the implementation of the system hardware. However the spatial resolution of the thick pinhole imaging is determined by the aperture of the pinhole, which can be reduced to improve the spatial resolution. In addition, this method for nondestructive detection will not be restricted by the source size.

Generally, performance of the imaging diagnostic system is calibrated with three main technical specifications: the modulation transfer function (MTF) [4], the noise power spectrum (NPS) [5] [6], and the detective quantum efficiency (DQE) [7] [8]. MTF is a universally accepted standard to calibrate the spatial resolution of the system. NPS, reflecting of the effects of the noise on the image quality, could present the difference of the image quality under different conditions of frequency spectra. DQE is used to reflect the detecting efficiency of the imaging diagnostic system to the $\gamma$-ray transmittance from the radiation source. Among the above-mentioned three specifications, there should be certain inherent relationships. In the case of the diagnostics of the high energy radiation sources, due to the relatively intense penetration effects of the $\gamma$-rays, the edge method becomes the major method to determine the MTF, which is represented by the Fourier Transform of the linear spread function (LSF). As for the NPS, it is resulted from the Fourier Transform of the image under the conditions of the flat field effects. The noise power spectra are different under different irradiation conditions. Another performance of the NPS is that it could be used to provide other parameters such as the signal-noise ratio $(S N R)$. Finally in dealing with the $\mathrm{DQE}$, it is related with such parameters as the detecting efficiency and quantum gain during the signal transmission [9]. The fluorescence gain of the scintillator is more than $10^{3}$ photon/MeV [10]. At the same time, the quantum gain of the $M C P$ imaging intensifier is also higher than $10^{3}$ photon/photon. Thus, the quantum gain becomes an important factor affecting the image quality of the high-gain imaging diagnostic system.

In this paper, relationships among various parameters of the gamma-rays camera, $M T F$, NPS and DOE, are researched based on thorough analysis of the 
$\gamma$-rays camera. Meanwhile, the effects of these parameters upon the image quality are evaluated and studied experimentally.

\section{Basic Principles of Pinhole Imaging with Gamma Rays}

The schematic diagram of the basic principle of the thick pinhole imaging with the $\gamma$-rays is given in Figure 1. The process could roughly be interpreted as follows. Firstly, the radiation source is imaged onto the imaging plane (YAG) by $\gamma$-rays. YAG crystal is used to convert the $\gamma$-rays into the fluorescence image. Then the fluorescence image is imaged onto the incident plane of the $M C P$ image intensifier through the optical imaging system and conversion system. Finally, the intensified image is recorded by $C C D$ device, and the $M C P$ and $C C D$ are coupled with fiber plate. Thus, the major two parts of the system are $\gamma$-rays imagines system and the image recording system. Besides, a copper reflector with an efficiency of over $95 \%$ is placed in a $45^{\circ}$ angle with $\gamma$-rays direction to reflect the fluorescence image, as well to avoid direct $\gamma$-rays irradiation onto the $M C P$ image intensifier and $C C D$ camera. The specially developed imaging system could provide an amplification factor of 5:1 and a light collection efficiency of over $95 \%$. The $M C P$ image intensifier was manufactured by Proxitronic Co. Ltd. with a spatial resolution of over $37 \mathrm{lp} / \mathrm{mm}$. The amplification factor of the fiber plate used to couple the $M C P$ and $C C D$ is $1.5: 1$, providing a stable and reliable recording system.

\section{Theoretical Modeling}

\subsection{Pinhole Imaging System}

Suppose the detection efficiency of the $\gamma$-rays in the scintillator is $\Phi$, and that the interactions of the $\gamma$-rays and the scintillator are abiding by the Gaussian distribution, then the fluctuated noise distribution induced by the detection efficiency would be $\sqrt{\Phi}$, and accordingly the $S N R$ of the signal input would be $S N R=\sqrt{\Phi}$. Another major factor to affect the quantum efficiency is the commonly called Swank coefficient, the energy distribution of the secondary electrons induced by the interactions of the $\gamma$-rays and the scintillator. Due to the fact that the $\gamma$-rays camera used in the experiment has a high gain and good detection efficiency for single particle, the effects of Swank coefficient on the image quality was excluded during our data analysis.

According to the pinhole imaging principles, the detection efficiency on the scintillator imaging plane after the thick pinhole imaging with the $\gamma$-rays could be given in the following equation [11]:

$$
\Phi=\phi \cdot \frac{\pi}{4}\left(\frac{D}{L}\right)^{2} \cdot \frac{1}{M^{2}} \cdot(1-\exp (-\mu t))
$$

where: $\phi$ is the output intensity of the $\gamma$-rays from the radiation source; $D$ is the pinhole diameter; $L$ is the object distance, $M$ is the amplification factor; $t$ is the scintillator thickness; $\mu$ is scintillator linear attenuation coefficient. Substitute the 


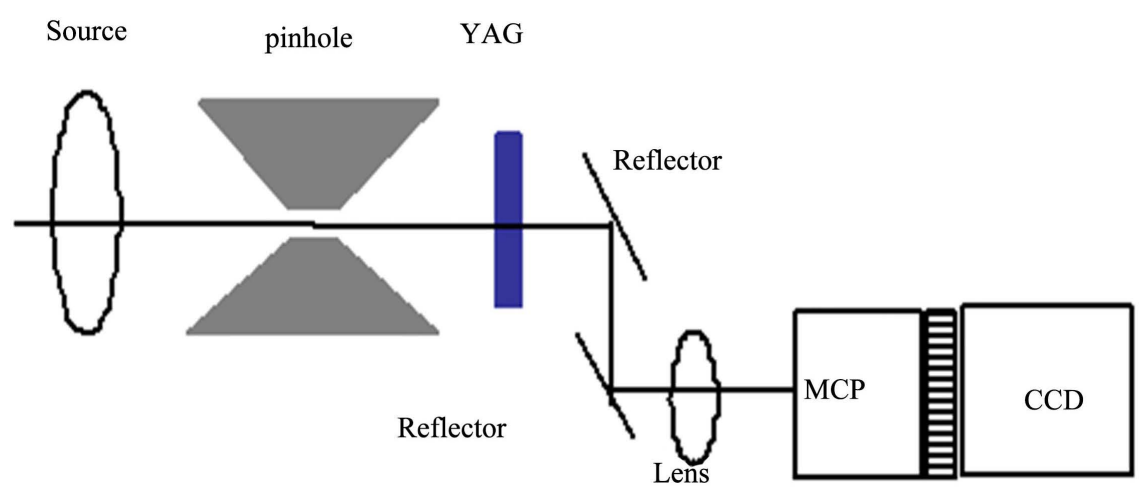

Figure 1. The schematic diagram of the thick pinhole imaging with $\gamma$-rays.

$S N R$ into Equation (1), we have the following equation to express the relationship between the $S N R$ and the radiation source intensity:

$$
\phi=\frac{4(L M)^{2}}{\pi D^{2}(1-\exp (-\mu t))} S N R_{i n}^{2}
$$

Again, based on the definition $D Q E$ [12], we would have the following equation:

$$
D Q E(f)=\left(\frac{S N R_{\text {out }}}{S N R_{\text {in }}}\right)^{2}
$$

Substitute Equation (2) into Equation (3), the relationships among the radiation source intensity, $D Q E$ and $S N R$ are obtained as follows:

$$
\phi=\frac{4(L M)^{2}}{\pi D^{2}(1-\exp (-\mu t))} \cdot \frac{S N R_{\text {out }}^{2}}{D Q E(f)}
$$

As indicated in Equation (4), in dealing with a given imaging diagnostic system, a radiation source with a corresponding intensity is necessary for a desired image quality to fulfill the experimental requirements or to get an ideal image under a certain $S N R$ condition.

\subsection{DQE of Image Recording System}

The image of the radiation source is recorded in $C C D$, where, the signal intensity for each of the pixels is $N p=\Phi \cdot A d / n p$, where $A d$ is the corresponding area on the scintillator of $n p$ pixels. The system gain is related to $\operatorname{MTF}(f)$, and the output signal could be given in the following:

$$
S_{\text {out }}=G \cdot \operatorname{MTF}(f) \cdot N_{p}
$$

where, $G$ is the signal gain with a 0 spatial frequency. The $C C D$ signal output is constrained by the NPS of the system. And the noises of the $C C D$ recording system are coming from three sources. The first source is the detection uncertainty of the scintillator detector. For example, in the case of the Gaussian distribution, where the detection uncertainty is $\sqrt{N_{p}}$, and the gained noise distribution of the detecting signals in the transmission system is $G M T F(f)$, the signal noise 
output from the $C C D$ device should be $\sigma n n=G M T F(f) \cdot \sqrt{N_{p}}$. The second source is the uncertainty of the transmission gain of the $\gamma$-rays $\sqrt{G}$. Namely, if the detection uncertainty of the $\gamma$-rays for each of the pixels is $\sqrt{N_{p}}$, the corresponding noise would be $\sigma_{n p}=\sqrt{G \cdot N_{p}}$. Finally, the third source is the dark current in the recording system, interpreted as $\sigma_{n d}=n r \cdot t e$ ( $n r$ is the dark charges in a unit time and te is the exposure time). Thus, the corresponding noise distribution for each of the pixels could be obtained as follows:

$$
\begin{gathered}
N P S(f)=\sqrt{\sigma_{n n}^{2}+\sigma_{n p}^{2}+\sigma_{n d}^{2}} \\
N P S(f)=\sqrt{N_{p} \cdot\left(G^{2} \cdot M T F^{2}(f)+G\right)+\left(n_{r} t_{e}\right)^{2}}
\end{gathered}
$$

And the $S N R$ of the $C C D$ output signals is given in the flowing:

$$
S N R_{\text {out }}(f)=\frac{G \cdot \operatorname{MTF}(f) \cdot N_{p}}{\sqrt{N_{p} \cdot\left(G^{2} \cdot M T F^{2}(f)+G\right)+\left(n_{r} t_{e}\right)^{2}}}
$$

Suppose the input signals are in the Gaussian distribution, and the $S N R$ of the input signals could be as follows:

$$
S N R_{i n}=\sqrt{\Phi \cdot A_{d} / n_{p}}=\sqrt{N_{p}}
$$

Then, according to Equation (3), the $D Q E$ for the $C C D$ imaging recording system should be:

$$
D Q E(f)=\frac{G^{2} \cdot M T F^{2}(f) \cdot N_{p}}{N_{p} \cdot\left(G^{2} \cdot M T F^{2}(f)+G\right)+\left(n_{r} t_{e}\right)^{2}}
$$

Again, in the case of an ideal scientific-level $C C D$ whose dark charges could be reasonably negligible, Equation (10) could be well rewritten into:

$$
D Q E(f)=\frac{G \cdot M T F^{2}(f)}{\left(G \cdot M T F^{2}(f)+1\right)}
$$

Substitute Equation (11) into Equation (4), the relationships among the radiation source intensity, $M T F$, quantum gain and output $S N R$ could be given in the following:

$$
\phi=\frac{4(L M)^{2}}{\pi D^{2}(1-\exp (-\mu t))} \cdot \frac{S N R_{\text {out }}^{2} \cdot\left(G \cdot M T F^{2}(f)+1\right)}{G \cdot M T F^{2}(f)}
$$

Based on the careful deduction mentioned above, the relationships among the radiation source intensity, $S N R$, quantum gain and $M T F$ could be well established. And in dealing with a given system and image $S N R$, the necessary intensity of the radiation source could also be determined.

\section{Analyses on Major Parameters}

\subsection{Quantum Gain $(G)$}

The quantum gain of the detector, $G$, depends on the system $\operatorname{MTF}(f)$, whose value in different spatial frequencies could be expressed as $G \cdot M T F(f)$. When $f=$ 
$0, M T F(0)=1$. And the quantum gain is decreased gradually with the increasing spatial frequency. While the $M T F$ is related with the gains of multiple components of the system, including the $\gamma$-ray-fluorescence convertor, the optical imaging system, the $M C P$ image intensifier and the $C C D$ device, etc. however, in the actual calculations, the gain at $f=0$ is common used as the standard.

The quantum gain mainly consists of the flowing components: $\Phi$ (the acting efficiency between the $\gamma$-rays and the scintillator); G $\gamma$ (the number of the fluorescence lights induced by each of the activated detected particle); $\varepsilon_{l}$ (the fluorescence efficiency of the imaging system); $\varepsilon M C P$ (the $D Q E$ of the $M C P$ image intensifier); MMCP (the gain of the $M C P$ image intensifier); $\varepsilon t a p$ (the penetration rate between the $M C P$ fluorescence screen and $C C D$ ); $\varepsilon C C D$ (the quantum efficiency of $C C D$ device). Other factors that affect the quantum gain include parameters of the simulated conversion systems inside the $C C D$ device. Finally, the quantum gain of the system could be given in the following equation:

$$
G=\Phi G_{\gamma} \varepsilon_{l} \varepsilon_{M C P} M_{M C p} \varepsilon_{t a p} \varepsilon_{C C D}
$$

The following are some specifications of the equipments or devices used in the experiment. The quantum gain of $Y A G$ crystal is about over $1.5 \times 103 / \mathrm{MeV}$. The optical imaging system was deliberately processed with a fluorescence efficiency of over $1 \%$. The $M C P$ image intensifier with conductive matrix could provide a quantum efficiency of about $45 \%$. The coupling efficiency of the fiber plat for $M C P$ image intensifier and $C C D$ device are about $70 \%$. The quantum efficiency of the backward photosensitive CCD device is more than $90 \%$. The scientific-level $C C D$ could provide a 16bit transmission in a digital conversion mode of $6 \mathrm{e} / \mathrm{ADU}$. After all, the image recording system, as a whole, could provide a satisfactory performance in the single particle detection of $\gamma$-rays, and could be used for the image diagnostics of the weak radiation sources.

\subsection{Modulation Transfer Function (MTF)}

As mentioned above, the system consists of a thick pinhole, $Y A G$ crystal, $M C P$ image intensifier, $C C D$ camera and other devices. According to the law of the $M T F$ transference, the system $M T F$ could be given in the following equation:

$\operatorname{MTF}(f)=\operatorname{MTF}(f)_{P} \cdot \operatorname{MTF}(f)_{Y A G} \cdot \operatorname{MTF}\left(f \cdot M_{M C P}\right)_{M C P} \cdot \operatorname{MTF}\left(f \cdot M_{C C D}\right)_{C C D}(14)$

In the calculation, the spatial resolution at the scintillator would be set up as the standard, while the MTF values of other devices should be converted into the spatial resolution at the scintillator in accordance with the amplification factor of the optical imaging system. For example, with a $M C P$ image intensifier of $40 \mathrm{lp} / \mathrm{mm}$ in the maximal spatial resolution, if the amplification factor of the optical imaging system is 0.2 , the corresponding spatial resolution at the scintillator should be about $8 \mathrm{lp} / \mathrm{mm}$. In this extent, the amplification factor of the imaging system should have relatively considerable effects on the MTFin the image transmission. For different device, the measurement of $M T F$ is also dif- 
ferent. For example, the $M T F$ measurement of $M C P$ image intensifier and $C C D$ device could be made with slit method and point source method, by which the $M T F$ could be obtained from $L S F$. Meanwhile, for the YAG scintillator under the $\gamma$-rays irradiation, the $M T F$ could be obtained mainly with edge method. Notably, there are some other factors to affect the MTF that could not be negligible, such as the dispersion effects of the secondary $\gamma$-rays and the fluorescent lights in the scintillator.

Besides the experimental measurement, the $M T F$ of the detector could also be provided by mathematical algorithm. For example, generally, the following could be obtained from calculation, such as the effects of the fiber plate structure on the $M C P$ image intensifier, the effects of the $C C D$ pixel size on the image recording, the effects of the lens size on the optical spatial resolution, and the spatial resolution of the pinhole imaging system, etc. And for the separated devices, the $M T F$ could be obtained from the following equation:

$$
\operatorname{MTF}(f)=\frac{\sin (\pi f D)}{\pi f D}
$$

where: $f$ is the spatial resolution; $D$ is the size of any one of the following items, including the size of the pinhole aperture, the size of the $C C D$ pixel, the diameter of the lens, the spatial resolution of the fiber plate, etc. The $M T F$ of the image diagnostic system is the total sum of the MTFs of all the devices, which is given in Equation (14). As for the thick pinhole imaging system, except the $M T F$ of the pinhole imaging system, the MTFs of all the devices that compose the image system could be determined experimentally.

\subsection{Effects of $S N R$ on Image Quality}

In the radiation imaging diagnostics, in order to get the radiation image worthy of analysis especially the image reflecting the evolution of the spatial intensity, the output image should have to have a certain $S N R$. For example, in the case of a relatively intense radiation source, the output image is generally required to have a $S N R$ of bigger than 5 to contain the effects of the noise upon the image quality. Again, since the $S N R$ is mainly depending on the detection efficiency of the $\gamma$-rays for the scintillator, the scintillator with relatively bigger thickness is common used to provide better detection efficiency. This is the reason for the widely application of the fiber array scintillators in the ICF experiments. However, such schemes for a higher $S N R$ work but with a compromise of a lower spatial resolution, because the spatial resolution is limited by the fiber scintillator.

\section{Experiment and Measurement Data}

\subsection{Measurement of Background Noise}

The background noise of the system was measured in the beginning. During the measurement, the imaging system is placed in a hohlraum, where the background noise induced by the hot electrons was measured, and the detection effi- 
ciency on the scintillator of the cosmic $\gamma$-rays as well. The maximum signal of the system was 65,535 . The integral time for the measurement of the background noise was $1000 \mathrm{~s}$. And the average signal obtained was 2000, indicating a $S N R$ of 30 in comparison with the 65,535 saturated signals. Actually in our experiment, the recording time of the system in the radiographic imaging process is only $2 \mathrm{~ms}$, a so short time with little effect negligible on the image quality.

\subsection{Experimental Measurement of $M T F$}

With a cobalt radiation source, the edge method was to measure the system $M T F$. The radiation source was a $0.7 \mathrm{~cm} \times 45 \mathrm{~cm}$ rod, shielded with a lead slit collimator ( $5 \mathrm{~mm} \times 50 \mathrm{~mm}$ in size and $200 \mathrm{~mm}$ in thickness). In this way, the size of the radiation source was confined within a range $5 \mathrm{~mm}$ in width. A tungsten edge collimator (10 $\mathrm{cm}$ in thickness) was placed tightly contacting the scintillator to shield the $\gamma$-rays and to provide the edge image appropriate for analysis. Then, a laser beam about $2 \mathrm{~mm}$ in diameter was introduced as the fundamental beam, which would pass throng the collimator center and irradiate onto the scintillator perpendicularly. The tungsten collimator was placed on a rotator. The best edge image could be obtained by rotating the rotator and monitoring the edge images. With this image, the linear spread function $(L S F)$ could be obtained. And the $M T F$ could be available after Fourier transformation.

In the case of a thick pinhole $0.7 \mathrm{~mm}$ in aperture size, the relative intensities with different frequencies could be calculated according to Equation (15) (Figure 2). As shown, the maximal resolution of the image recording system was about $1.5 \mathrm{lp} / \mathrm{mm}$, basically same with that of the thick pinhole imaging system.

\subsection{Quantum Gain}

The quantum gain of the image diagnostic system is closely dependent on the performance and working state of the components. Since the quantum gain for each of the components is obtained under a given specific condition, relatively big uncertainly is inevitable in the evaluation of the total quantum gain of the system. Another way to calibrate the total quantum gain of the image recording system is to carry out experiment. In our experiment, the deliberately designed optical imaging system with relatively high light collection efficiency, could provide detection efficiency for single particle for the system. However, the detected signals of the single particles are different, due to the deposited energy of the secondary electrons in the scintillator and different transmission distance of the secondary electron. As seen in Figure 3 and Figure 4, the average intensity under lower radiation intensity is about 42 . At the same time, for every single particle, its occupation in the image would be 2 to 4 pixels. Thus, based preliminary evaluation, the total quantum gain for each of the activated $\gamma$-rays was about 150. Since the $M C P$ image intensifier has 10 tunable electric amplification factors, the quantum gain would also be different under the different electronic gain conditions. 


\subsection{Output SNR}

During the experiments, considerable measurements were made, including those of the $M T F$, quantum gain and the related noise power spectra. Based on the experiments, a relatively keen insight and deep understanding of the image characteristics were obtained. The minimal radiation intensity required for various $S N R$ conditions could be calculated in accordance with Equation (12). And the radiation source intensity varies with various frequencies. Thus, in order to get a radiation image appropriate for analysis, the $S N R$ is generally required to be higher than 5. Equation (12) was used for the calculation of the radiation intensities in various frequency conditions, whose results are given in Figure 5. As indicated in the results, a higher $S N R$ could be available with higher radiation source intensity.

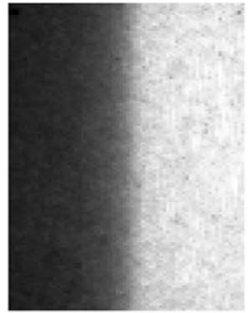

(a)

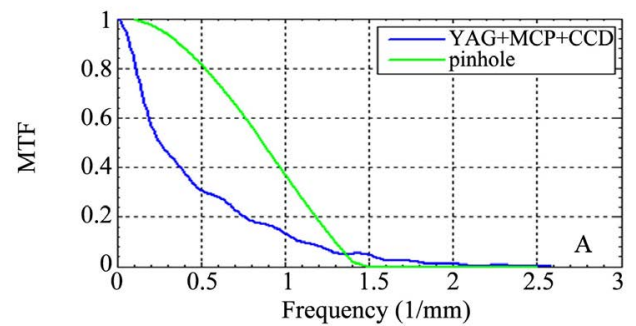

(b)

Figure 2. (a) The edge image measured with cobalt radiation source; (b) The $M T F$ of the pinhole imaging system experimentally measured.

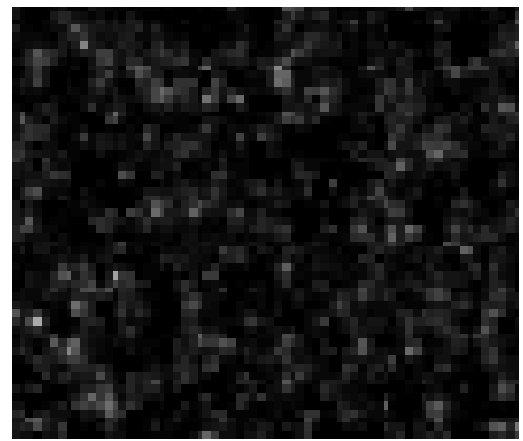

Figure 3. The radiation image under a single particle action.

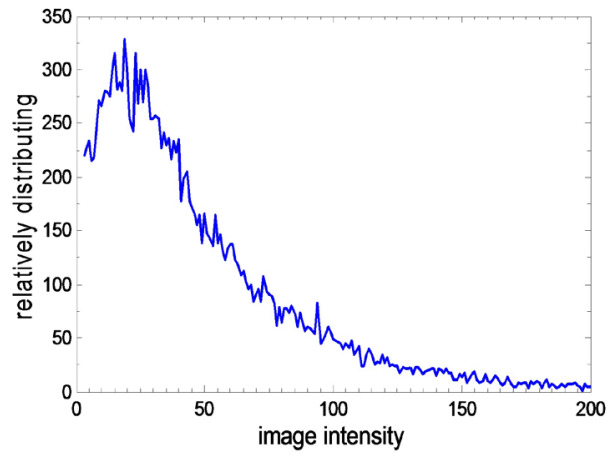

Figure 4. The schematic distribution of the quantum gain. 


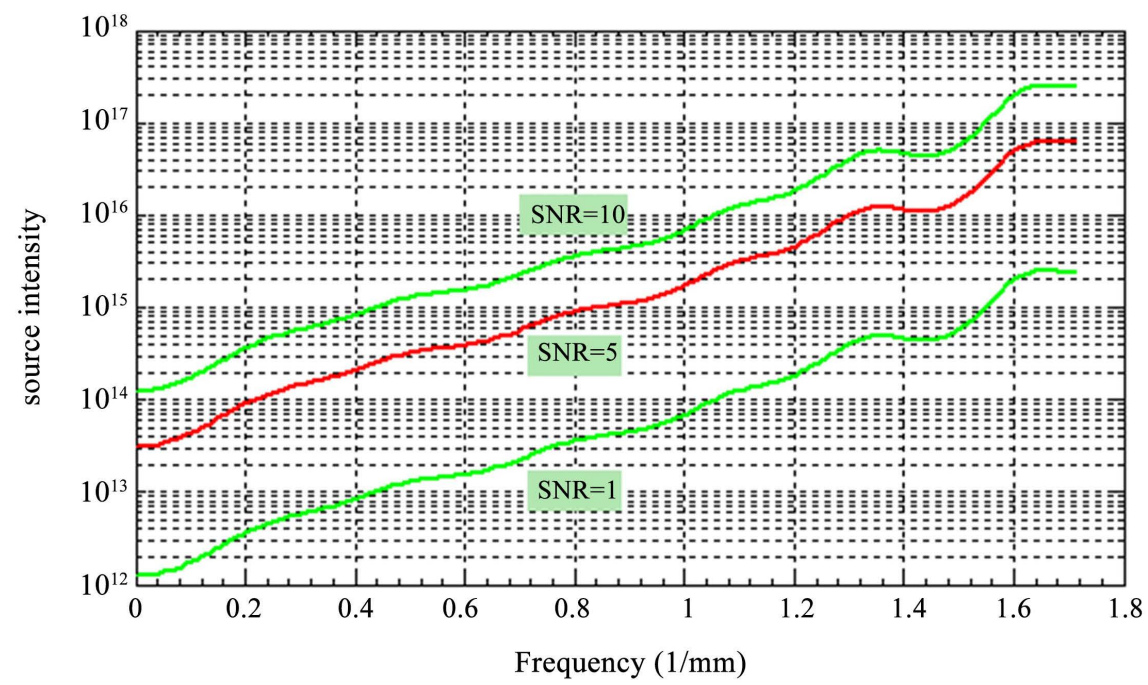

Figure 5. The schematic diagram of the relationship among the radiation source intensity, the $S N R$ and the spatial frequency.

\section{Data Analysis}

\subsection{Effects of $D Q E$ on Image Quality}

The $D Q E$ of the image diagnostic system is mainly dependent on the probability of the interaction between the $\gamma$-rays and the scintillator. The spurious speckle effects in the image are mainly due to the energy of the secondary electrons and fluorescent efficiency. And size of the speckles is determined by the electronic transmission distance (i.e. the fluorescent dispersion). Each of the interacting spot would be considered as a spot light source, and the total sum of the spots would reflect the image intensity. Due to the spurious speckle effects and their distribution, under a weak radiation condition, the image intensity would not be changed linearly with the change of the radiation intensity.

The deliberately designed optical imaging system is characterized in a high light collection efficiency, high $D Q E$ and optical gain in the optoelectronic system. The thermal noise of the electronic system is so little and could be neglected. The system could have good detection efficiency for a single photon to capture each of the fluorescent light induced by the interaction between the scintillator with the secondary electron. The quantum gain in the optimal conditions could be as high as about 150 . In dealing with maximal image intensity of about 65,535 , the dynamic range of the quantum gain of the imaging system was within about 437 . Thus the requirements for the image diagnostics with relatively small uncertainty could be well fulfilled.

\subsection{System $M T F$}

The image recording system mainly consists of the $Y A G$ scintillator, the $M C P$ image intensifier, $C C D$ camera and other devices. Each of the components has its own way for the MTF measurement. All the MTFs of the components were measured independently in accordance with the corresponding measurement 
method. And the $M T F$ of the system would be the total sum of the all the MTFs of the components. However, due to measurement conditions for the each of component varies from each other, the total MTF of the system would be obtained with big uncertainty. In this extent, the edge method could not be used for the direct measurement of the $M T F$ of the imaging system. In our experiment, the edge collimator was placed on the rotator to monitor the evolution of the image quality. Due to limited experimental conditions, the monitoring was made only for a range from $-5^{\circ}$ to $5^{\circ}$. During this process, image quality was found with little change, which should be another implication that the fluorescent dispersion should be a major cause for the image degradation. And the fluorescent dispersion is then composed of the dispersion effects in the scintillator and the defocusing effects of the optical imaging system. In order to get higher light collection efficiency, the spatial resolution of the optical imaging system has to be decreased. In this context, the spatial resolution might not be as good as expectation. As compensation, the high light collection efficiency could provide fairly good results in the image diagnostics especially in dealing with the weak radiation conditions with special requirements. The spatial resolution of the thick pinhole imaging system could not be measured in certain conditions, which could be obtained mainly based on the theoretical design and related theoretical calculation.

\section{Conclusion}

The principle of the using pinhole imaging to obtain the radiation source profile was introduced in this paper. The image diagnostic system consisted of a pinhole, a $Y A G$ scintillator, a optical imaging system, a $M C P$ image intensifier and a $C C D$ device. The relation of the signal transmission process, $D Q E, N P S$, quantum gain, $S N R$ of the system and the source intensity was analyzed. The main conclusions were as follows, 1 ) The $M T F$ at $10 \%$ of the system was about $1 \mathrm{LP} / \mathrm{mm} ; 2$ ) As the single-particle detection efficiency of the system, the stray speckle image would be observed under low radiation intensity, based on which the quantum gain of the system could be obtained as about 150;3) As well the single-particle detection efficiency, the effect of the secondary gamma ray and fluorescence to the spatial resolution would totally submerged in the quantum fluctuation caused by the stray speckle; 4) Based on the established relation of the $D Q E$, quantum gain, $S N R$ and the source intensity, the minimum source intensity required under different $S N R s$ was calculated.

\section{Conflicts of Interest}

The authors declare no conflicts of interest regarding the publication of this paper.

\section{References}

[1] Christensen, C.R. and Barnes, C.W. (2003) First Results of Pinhole Neutron Imag- 
ing for Inertial Confinement Fusion. Review of Scientific Instruments, 74, 2690-2694.

[2] Yates, G.J. and King, N.S.P. (1997) High-Frame-Rate Intensified Fast Optically Shuttered TV Cameras with Selected Imaging Applications. SPIE Conference Proceedings, Vol. 2273, 26-149.

[3] Vartsky, D. (2009) Signal and Noise Analysis in TRION-Time-Resolved Integrative Optical Fast Neutron Detector, Soreq NRC, Yavne 81800. Journal of instrument, 2, 1-18.

[4] Xiang, C.C. and Chen, X.H. (2010) MTF Measurement and Imaging Quality Evaluation of Digital Camera with Slanted-Edge Method. Proceedings of SPIE, Photonics Asia, Vol. 7849, Beijing, 5 November 2010, 78490A.

https://doi.org/10.1117/12.869937

[5] Park, H.-S. and Kim, H.-J. (2008) Measurements and Evaluation of the Image Noise Power Spectrum for Computed Radiography. 2008 IEEE Nuclear Science Symposium Conference Record, Dresden, 19-25 October 2008, 4378-4383. https://doi.org/10.1109/NSSMIC.2008.4774252

[6] Miévillea, F.A., Bolardbm G., et al. (2011) 3D Noise Power Spectrum Applied on Clinical MDCT Scanners: Effects of Reconstruction Algorithms and Reconstruction Filters. SPIE Medical Imaging, Vol. 7961, Orlando, 79610E.

[7] Lubinsky, A.R. and Zhao, W. (2008) Screen Optics Effects on DQE in Digital Radiography: Spatial Frequency Effects, Proceedings of SPIE, Medical Imaging 2008: Physics of Medical Imaging, Vol. 6913, San Diego, 18 March 2008, 69134F. https://doi.org/10.1117/12.772973

[8] Park, H.-S. and Kim, H.-J. (2010) Effects of Image Processing on the Detective Quantum Efficiency. SPIE Medical Imaging, Vol. 7622, San Diego, 23 March 2010, $76220 \mathrm{M}$

[9] Gang, G.J. and Zbijewski, W. (2012) Theoretical Framework for the Dual-Energy Cone-Beam CT Noise-Power Spectrum, NEQ, and Tasked-Based Detectability Index. Proceedings of SPIE, SPIE Medical Imaging, Vol. 8313, San Diego, 23 February 2012, 83131J. https://doi.org/10.1117/12.911817

[10] Mah, D. and Rawlinson, J.A. (1999) Detective Quantum Efficiency of an Amorphous Selenium Detector to Megavoltage Radiation. Physics in Medicine \& Biology, 44, 1369-1384. https://doi.org/10.1088/0031-9155/44/5/320

[11] Xie, H.W., Peng, T.P., Xu, Z.P. and Chen, F.X. (2011) Thick Pinhole Design Applied to High Energy $\gamma$-Ray Source Radiographic Diagnostics. Nuclear Electronics \& Detection Technology, 31, 143-147.

[12] Cremer, J.T., Piestrup, M.A. and Wu, X.Z. (2005) Fast and Thermal Neutron Radiography. Proceedings of SPIE, Optics and Photonics, Vol. 5923, San Diego, 21 September 2005, 59230A. https://doi.org/10.1117/12.624972 